\title{
Alfvén waves in the foreshock propagating upstream in the plasma rest frame: statistics from Cluster observations
}

\author{
Y. Narita ${ }^{1}$, K.-H. Glassmeier ${ }^{1,2}$, S. Schäfer ${ }^{1}$, U. Motschmann ${ }^{3}$, M. Fränz ${ }^{2}$, I. Dandouras ${ }^{4}$, K.-H. Fornaçon ${ }^{1}$, \\ E. Georgescu ${ }^{5}$, A. Korth ${ }^{2}$, H. Rème ${ }^{4}$, and I. Richter ${ }^{1}$ \\ ${ }^{1}$ Institut für Geophysik und Meteorologie, TU Braunschweig, Mendelssohnstr. 3, D-38106, Braunschweig, Germany \\ ${ }^{2}$ Max-Planck-Institut für Aeronomie, Max-Planck-Straße 2, D-37191 Katlenburg-Lindau, Germany \\ ${ }^{3}$ Institut für Theoretische Physik, TU Braunschweig, Mendelssohnstr. 3, D-38106, Braunschweig, Germany \\ ${ }^{4}$ CESR/CNRS, 9 Avenue du Colonel Roche, Toulouse Cedex 4, F-31028, France \\ ${ }^{5}$ Max-Planck-Institut für extraterrestrische Physik, Giessenbachstraße, D-85748 Garching, Germany
}

Received: 11 September 2003 - Revised: 4 February 2004 - Accepted: 18 February 2004 - Published: 14 July 2004

Part of Special Issue "Spatio-temporal analysis and multipoint measurements in space"

\begin{abstract}
We statistically study various properties of lowfrequency waves such as frequencies, wave numbers, phase velocities, and polarization in the plasma rest frame in the terrestrial foreshock. Using Cluster observations the wave telescope or $k$-filtering is applied to investigate wave numbers and rest frame frequencies. We find that most of the foreshock waves propagate upstream along the magnetic field at phase velocity close to the Alfvén velocity. We identify that frequencies are around $0.1 \times \Omega_{c p}$ and wave numbers are around $0.1 \times \Omega_{c p} / V_{A}$, where $\Omega_{c p}$ is the proton cyclotron frequency and $V_{A}$ is the Alfvén velocity. Our results confirm the conclusions drawn from ISEE observations and strongly support the existence of Alfvén waves in the foreshock.
\end{abstract}

Key words. Electromagnetics (wave propagation) - Interplanetary physics (planetary bow shocks) - Space plasma physics (waves and instabilities)

\section{Introduction}

Waves upstream of the Earth's bow shock have been widely studied since early observations in the 1960s. The existence of a foreshock is characteristic of collisionless plasma, making a contrast to shocks in ordinary, collisional gas dynamics. In a collisionless plasma charged particles can not only travel downstream across the shock along the magnetic field relatively easily but can also deviate upstream as they gain energies at the shock. The backstreaming particles, together with the plasma flowing downstream, form distribution func-

Correspondence to: Y. Narita

(y.narita@tu-bs.de) tions which are unstable to different types of waves at various frequencies (e.g. Burgess, 1997). The foreshock is thus a fascinating region for studying waves and instabilities.

Backstreaming ions and associated low-frequency waves were discovered by Asbridge et al. (1968), Greenstadt et al. (1968), and Fairfield (1969). They led to speculation that the waves were driven by backstreaming ions. These phenomena were studied in more detail using the ISEE dual spacecraft in various methods: morphology or wave form study, frequency analysis, polarization analysis, and estimating wavelengths. In the wave form study monochromatic wave packets at period of $30 \mathrm{~s}$ are dominating. The wave form study is summarized in Greenstadt et al. (1995). Different wave forms were associated with different classes of ion population, e.g. collimated ion beams, semicircular or crescent shaped ion distributions (intermediate distributions), and nearly isotropic diffuse ion distributions (Gosling et al., 1978). Paschmann (1979) showed that low-frequency waves were associated with the intermediate and diffuse ions. Theories of the foreshock waves have also been developed. Barnes (1970) studied an ion cyclotron instability and Fredericks (1975) studied gyrophase-bunched ions as generation mechanisms. The instability of the ion beam was studied in detail by, e.g. Gary (1991). He investigated the growth rate of the instability dependent on wave propagation direction and explained the association of waves with different ion distributions using the growth rate. Left-handed Alfvén waves are excited and propagate upstream in the rest frame in this theory. Sentman (1981) studied the non-resonant firehose instability produced by fast and dense backstreaming ion beams. The generated waves are right-handed and compressional, and propagate downstream in the rest frame in this theory. 


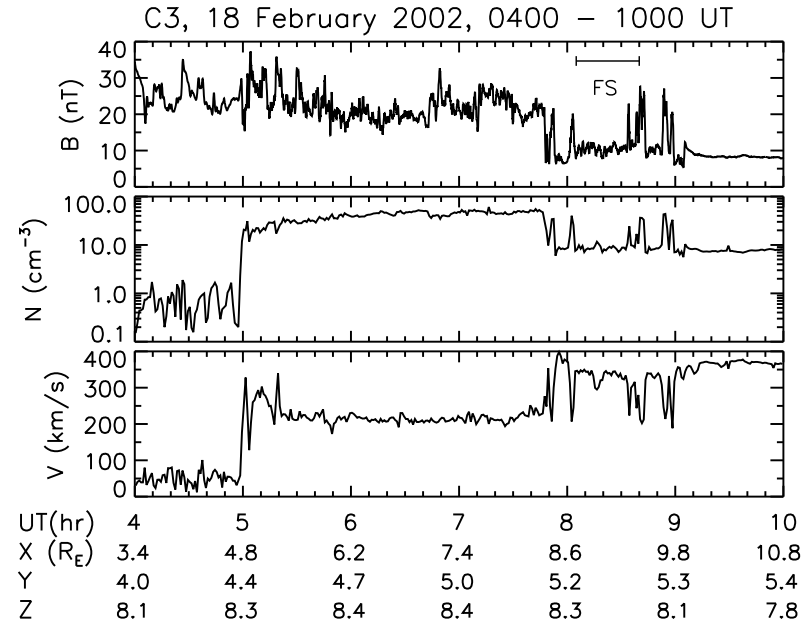

Fig. 1. Measurements of the magnitude of magnetic field from FGM, ion density and bulk velocity from CIS-HIA made by Cluster 3 from 04:00 to 10:00 UT on 18 February 2002. "FS" between 08:05 and 08:40 UT stands for an interval of the foreshock wave observation.

From an observational point of view, one of the most difficult problems was to investigate wavelengths or wave numbers. Information about spatial structures cannot be easily extracted from spacecraft data. In order to analyze waves in a proper frame of reference - the plasma rest frame investigation of wave numbers is inevitable. When a background flow exists, frequencies are changed by the Doppler shift: $\omega_{s / c}=\omega_{\text {rest }}+\boldsymbol{k} \cdot \boldsymbol{V}_{s w}$, where $\omega_{s / c}, \omega_{\text {rest }}, \boldsymbol{k}$, and $\boldsymbol{V}_{s w}$ represent angular frequency in the spacecraft frame and in the plasma rest frame, wave number vector, and background plasma flow velocity, respectively. There were, despite the general difficulty, opportunities to achieve this: Hoppe et al. (1981) and Hoppe and Russell (1983) demonstrated several cases from ISEE data in which wavelengths $\sim 1 R_{E}$ $\left(1 R_{E}=6370 \mathrm{~km}\right)$ and rest frame frequencies $\sim 0.1 \times \Omega_{c p}$ ( $\Omega_{c p}$ is the proton cyclotron frequency) were successfully identified. Le and Russell (1990) also presented that the coherence lengths of the waves were of the same order. When the flow velocity is opposite to the wave propagation direction and larger than the propagation speed, the Doppler shift exceeds the rest frame frequency and the waves propagate in the flow direction. In the case of the foreshock, waves propagating upstream may travel downstream because of the large solar wind velocity. Russell (1971) discussed that the foreshock waves were left-hand polarized in the spacecraft frame but intrinsically right-hand polarized, resulting from a reversal of phase speed direction. Such waves should have phase velocities in the plasma rest frame less than the solar wind velocity. Applying the wave analysis method for dual spacecraft data, Dudok de Wit et al. (1995), Balikhin et al. (1997a, b) presented experimental dispersion relations. They combined wave numbers projected into the spacecraft separation line with wave propagation direction derived from the minimum variance analysis.
The Cluster mission (Escoubet et al., 2001) has a potential to resolve spatial structure in three dimensions and allows one to investigate the wave numbers and the rest frame frequencies. Several interesting results have already been presented. Different types of waves, Alfvénic and fast magnetosonic, were identified which agreed well with the results from the ISEE observations (Eastwood et al., 2002, 2003). The wave dispersion relation can also be investigated using Cluster data. As demonstrated by Narita et al. (2003) an experimental dispersion relation was derived, which was in good agreement with the dispersion relation calculated for the beam plasma instability.

In this paper we briefly report on our study of the foreshock waves. The waves are analyzed statistically using Cluster data for the first time in the plasma rest frame.

Magnetic field data of 1- and 4-s resolution from the four identical fluxgate magnetometers (FGM, see Balogh et al., 2001 for instrumentation) on board Cluster are extensively used and the wave telescope or $k$-filtering technique developed by Pinçon and Lefeuvre (1991), Motschmann et al. (1995, 1996), and Glassmeier et al. (2001) is applied to obtain the wave numbers. Ion density and velocity data from Cluster Ion Spectrometry, Hot Ion Analyzer (CIS-HIA, Rème et al., 2001) from Cluster 3 are also used to calculate the Alfvén velocities and the Doppler shifts. We first present a case study in Sect. 2 and introduce our analysis method. We then present the statistical study in Sect. 3. In Sect. 4 we summarize and discuss our results.

\section{Case study}

We present a case study and explain our analysis method to obtain the frequency $\omega_{\text {rest }}$, the wave number $\boldsymbol{k}$, the phase velocity $\boldsymbol{V}_{p h(\text { rest })}$, and the ellipticity of polarization $\epsilon$. Figure 1 displays the magnitude of magnetic field, the ion density, and the ion bulk velocity in the time domain measured by Cluster 3 from 04:00 to 10:00 UT on 18 February 2002. The foreshock waves in this event exhibit the clearest example in wave power spectra and typical features which will be drawn in the statistical study. The spacecraft separation is as small as $100 \mathrm{~km}$ and the time series plots of magnetic field display almost the same results among different spacecraft. Cluster observes the dayside northern magnetosphere (04:00 05:00 UT), the magnetosheath (05:00-08:00 UT), and the upstream solar wind region (08:00-10:00 UT) with several shock crossings. The angle between the upstream magnetic field and the shock normal determined from the method of coplanarity theorem (e.g. Schwartz, 1998) is $2.6^{\circ}$ for Cluster 3 FGM data. In the upstream region moderately active fluctuation of the magnetic field is observed between 08:00 and 09:00 UT, with the average summed component of normalized magnetic field variance (for 4-s data) as

$\left\langle\sigma^{2}\right\rangle=\left\langle\frac{\left|\delta B_{x}\right|^{2}+\left|\delta B_{y}\right|^{2}+\left|\delta B_{z}\right|^{2}}{|B|^{2}}\right\rangle \sim 0.365$ 
whereas the fluctuation is quiet after 09:00 UT. We take the interval 08:05-08:40 UT for the case study of the foreshock waves. The mean value of interplanetary magnetic field (IMF) is $\boldsymbol{B}=(8.4,-4.1,2.5) \mathrm{nT}$ in GSE and the IMF cone angle (angle between the magnetic field direction and the $\mathrm{x}$-axis) is $29.8^{\circ}$. Our analysis method is as follows. First, the geometrical configuration of the foreshock observation is investigated. Then the dominant frequency in the spacecraft frame is identified from the power spectrum and the wave number is identified using the wave telescope. The Doppler shift is corrected to derive the rest frame frequency and the phase velocity. The ellipticity of polarization is also investigated using quasi-monochromatic wave theory.

The foreshock is generally connected to the shock by the magnetic field. We investigate the geometrical configuration for Cluster 3 which provides us with both FGM and CISHIA data and ask whether the observation is made in the foreshock region or not. We assume that CIS-HIA data from Cluster 3 are valid for other spacecraft in the wave analysis later. For simplicity we adopt an empirical, parabolic bow shock model

$X=a_{s}-b_{s}\left(Y^{2}+Z^{2}\right)$,

where $a_{s}$ is the bow shock standoff distance in unit of $R_{E}$ from the Earth and $b_{s}$ is the flaring parameter in unit of $R_{E}^{-1}$ (e.g. Merka et al., 2003). The bow shock is well represented by a paraboloid for GSE- $X$ larger than $-40 R_{E}$ (Cairns et al., 1995). The coordinate system is aberrated so that the direction of the $\mathrm{x}$-axis is opposite to the solar wind velocity $\boldsymbol{V}_{s w}$ and the $x y$ plane is made by $\boldsymbol{V}_{s w}$ and the IMF orientation (we call the $x y$ plane the VB plane). The IMF angle $\arctan \left(B_{y} / B_{x}\right)$ in the VB plane is $47.6^{\circ}$. The solar wind dynamic pressure and the fast magnetosonic Mach number calculated from Cluster 3 data are applied to obtain $a_{s}$ and $b_{s}$ using the relations

$a_{s}=a_{m p}\left[1+1.1 \frac{(\gamma-1) M^{2}+2}{(\gamma+1)\left(M^{2}-1\right)}\right]$

$b_{s}=0.0223\left(\frac{P_{s w}}{1.8}\right)^{\frac{1}{6}} \quad R_{E}^{-1}$,

where $a_{m p}$ is the magneopause standoff distance, $\gamma$ is the effective ratio of specific heats and assumed to be $5 / 3, M$ is the fast magnetosonic Mach number (e.g. Russell, 1985), $P_{s w}$ is the solar wind dynamic pressure in unit of nPa. Equations (3) and (4) were proposed by Farris and Russell (1994) and Cairns et al. (1995), respectively. $a_{m p}$ is calculated for the magnetopause model of Shue et al. (1997). We obtain $a_{s}=14.0 R_{E}$ and $b_{s}=2.25 \times 10^{-2} R_{E}^{-1}$. Now consider the equation

$\boldsymbol{r}=\boldsymbol{r}_{0}+D \boldsymbol{e}_{B}$

which relates the intersection of the IMF line connected to the spacecraft at the shock $\boldsymbol{r}$ to the spacecraft position $\boldsymbol{r}_{0}=\left(x_{0}, y_{0}, z_{0}\right)$ and the distance between them $D$ (see

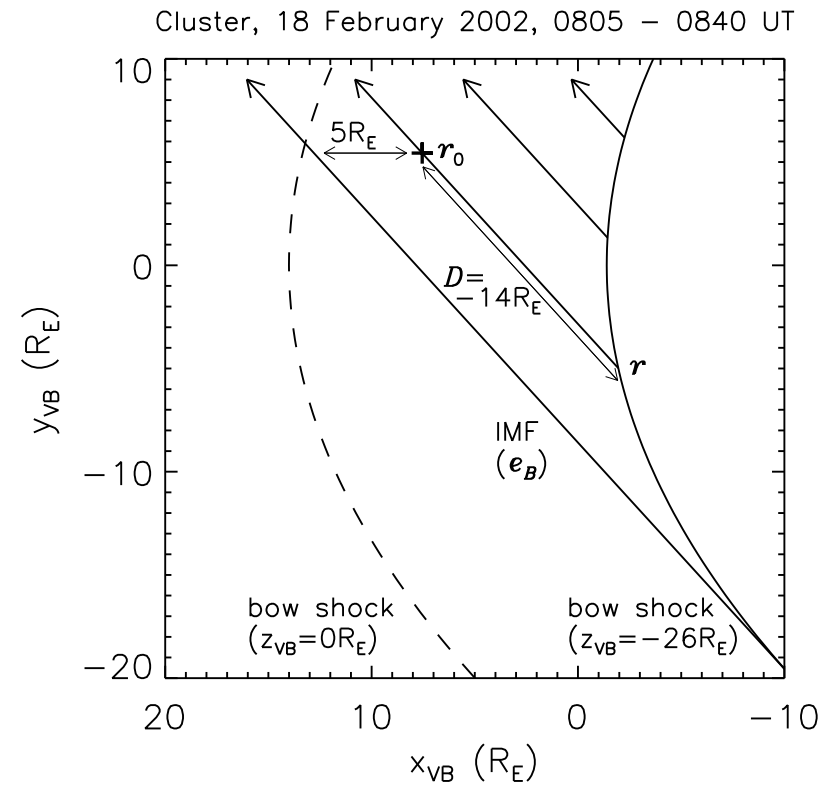

Fig. 2. Geometrical configuration of the foreshock wave observation between 08:05 and 08:40 UT on 18 February 2002. The x-axis is aberrated to the solar wind direction. The $x y$ plane is made by the solar wind and IMF direction, translated in the $z$ direction to the location of Cluster 3 at $z=-26 R_{E}$. Bow shock at $z=0 R_{E}$ projected into $z=-26 R_{E}$ is also present. $\boldsymbol{r}_{0}, \boldsymbol{r}, D$ and $\boldsymbol{e}_{B}$ represent Cluster 3 location, the intersection of IMF line connected to the spacecraft at the bow shock, the distance between $\boldsymbol{r}_{0}$ and $\boldsymbol{r}$, and the unit vector of IMF orientation, respectively.

Fig. 2). $\boldsymbol{e}_{B}=\left(e_{x}, e_{y}, e_{z}\right)$ is a unit vector of IMF and it is assumed that there is no change in mangnetic field topology. $D$ may take negative values, depending on the direction of IMF and the spacecraft position. Combining Eq. (2) with Eq. (5), one obtains a quadratic equation for $D$

$$
\begin{gathered}
b_{s}\left(e_{y}^{2}+e_{z}^{2}\right) D^{2}+\left[e_{x}+2 b_{s}\left(y_{0} e_{y}+z_{0} e_{z}\right)\right] D \\
\cdot \quad+\left[x_{0}-a_{s}+b_{s}\left(y_{0} e_{y}+z_{0} e_{z}\right)\right]=0
\end{gathered}
$$

Real solutions for $D$ represent the spacecraft located in the foreshock region. We obtain $D=-14.1 R_{E}$. The distance between tangential magnetic field line to the shock and the spacecraft along $\boldsymbol{V}_{s w}$ is $5.2 R_{E}$. Figure 2 displays a sketch of the result in the VB plane translated in $z$ direction to the spacecraft position at $z_{V B}=-26 R_{E}$.

Figure 3 displays the power spectrum of the magnetic field fluctuation derived from the Fast Fourier Transform (FFT) for Cluster 3. A peak in power is identified at frequency $44.9 \mathrm{mHz}$ (period of $22 \mathrm{~s}$ ). Power spectra derived from the other Cluster spacecraft exhibit the same result. The wave number at this frequency is investigated using the wave telescope. This technique allows one to calculate the wave power in the $k$-space (wave number space) from multispacecraft magnetic field data (Pinçon and Lefeuvre, 1991; Motschmann et al., 1995, 1996; Pinçon and Motschmann, 1998; Glassmeier et al., 2001). An example of the wave 


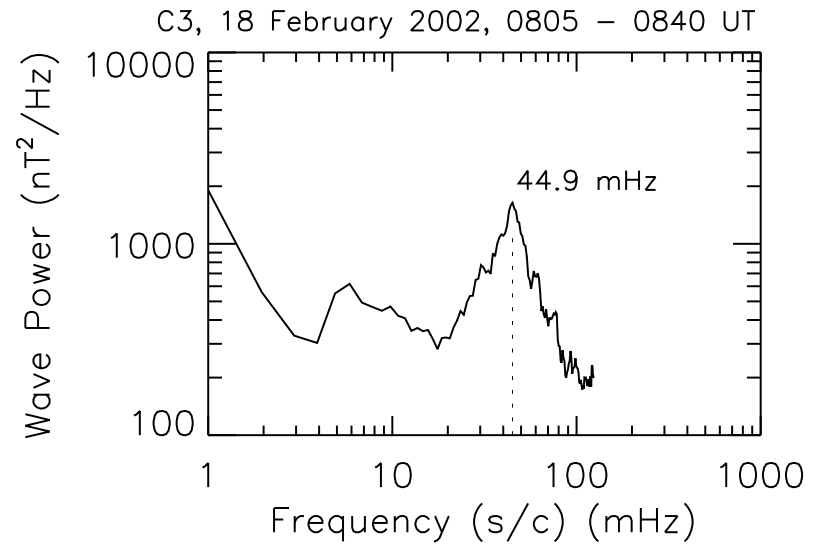

Fig. 3. Power spectrum in frequency domain in the spacecraft frame for the magnetic field data from Cluster 3 for the same interval as Fig. 2.

power derived from the wave telescope is displayed in Fig. 4. A sharp peak is found in the direction almost anti-parallel to the magnetic field. We identify this position as the wave number associated with the given frequency and obtain $\left(k_{\|}\right.$, $\left.k_{\perp}\right)=\left(-1.4 \times 10^{-3},-0.4 \times 10^{-3}\right) \mathrm{km}^{-1}$ (here $k_{\perp}$ is in the direction of $\boldsymbol{V}_{s w}$ projected into the perpendicular plane to the magnetic field $)$ and $\left(k_{x}, k_{y}, k_{z}\right)=\left(-1.1 \times 10^{-3}, 0.6 \times 10^{-3}\right.$, $\left.-0.7 \times 10^{-3}\right) \mathrm{km}^{-1}$ in GSE. The magnitude of the wave number is $1.47 \times 10^{-3} \mathrm{~km}^{-1}$ (wavelength $4284 \mathrm{~km}$ ) and the angle from the magnetic field $\theta_{k B}=163^{\circ}$.

The Doppler shift is corrected and the rest frame frequency is calculated using the relation $\omega_{\text {rest }}=\omega_{s / c}-\boldsymbol{k} \cdot \boldsymbol{V}_{s w}$, where $\omega_{s / c}=44.9 \times 2 \pi \mathrm{mHz}, \boldsymbol{V}_{s w}=(-317.4,55.8,17.8) \mathrm{km} / \mathrm{s}$, and $\boldsymbol{k}$ as above. We obtain $\omega_{\text {rest }}=-89.2 \mathrm{mHz}$. The physical meaning of the negative frequency becomes clear when discussing the phase velocity. The phase velocity is defined as $V_{p h}=\omega / k$ or $\boldsymbol{V}_{p h}=\omega \boldsymbol{k} /|k|^{2}$ in vectorial expression. In the spacecraft frame $\boldsymbol{V}_{p h(s / c)}=(-144.3,83.3,-96.2) \mathrm{km} / \mathrm{s}$ in GSE with the magnitude $192.4 \mathrm{~km} / \mathrm{s}$ and in the plasma rest frame $\boldsymbol{V}_{p h(r e s t)}=(45.6,-26.3,30.4) \mathrm{km} / \mathrm{s}$ with the magnitude $60.8 \mathrm{~km} / \mathrm{s}$. Thus, the direction of phase velocity is reversed. In the $x$ component, for example, the wave propagates in the anti-sunward direction in the spacecraft frame but in the sunward direction in the plasma rest frame. In other words, the wave propagates downstream because the solar wind velocity is larger than the rest frame phase velocity. To avoid the usage of negative frequencies we change the signs of $\omega$ and $\boldsymbol{k}$ without loss of generality. This keeps the phase velocity unchanged but changes the representation of the sense of the polarization from right-hand to left-hand representation and vice versa. We also change the propagation angle $\theta_{k B}$ into $180^{\circ}-\theta_{k B}$ to agree with the change of sign of $\boldsymbol{k}$. As a result, the propagation angle from the Sun-to-Earth direction is $41^{\circ}$ in the spacecraft frame and $131^{\circ}$ in the plasma rest frame. The normalized frequency is $\omega_{\text {rest }} / \Omega_{c p}=0.096$ and the normalized wave number is $k V_{A} / \Omega_{c p}=0.102$, where $\Omega_{c p}=0.931 \mathrm{~Hz}$ and $V_{A}=64.9 \mathrm{~km} / \mathrm{s}$ (Alfvén velocity).

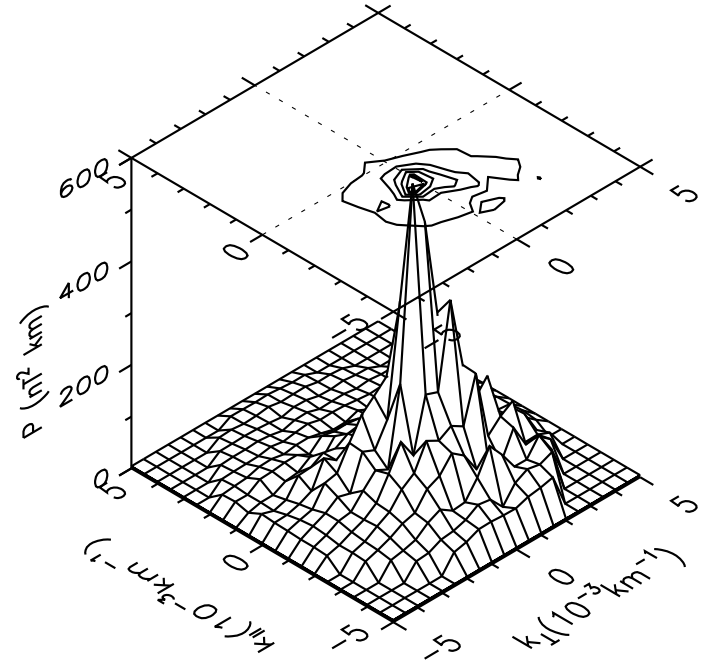

Cluster, 18 February 2002, 0805 - 0840 UT, 44.9 mHz

Fig. 4. Wave power in $k$-space made by the wave telescope and the magnetic field data from all four Cluster spacecraft for the same interval as Fig. 2. $k_{\perp}$ is in the same direction as ion bulk velocity projected into the perpendicular plane to the magnetic field.

Polarization is also investigated using the principles of optics as applied to quasi-monochromatic wave theory. The cross spectral density matrix

$G_{i j}(f)=\lim _{T \rightarrow \infty} \frac{2}{T} E\left[B_{i}^{*}(f, T) B_{j}(f, T)\right]$

is calculated (Bendat and Piersol, 1980; Born and Wolf, 1980). $E$ denotes the operation of expectation (ensemble average) and indices $i$ and $j$ run over $x, y$ and $z$ component. $B_{i}(f, T)$ is the finite Fourier transform of magnetic field at frequency $f$ over record length $\mathrm{T}$ and the asterisk means a complex conjugate. The polarization plane is then identified by finding eigenvalues and eigenvectors of $G_{i j}$, i.e. we search for directions of the principal and second principal variance. The ellipticity $\epsilon$ or ratio of minor to major axis is defined by

$\epsilon=\tan \beta$

and the sense of the polarization by the sign of $\beta$. The angle $\beta$ can be obtained from the spectral density matrix (e.g. Fowler et al., 1967; Born and Wolf, 1980):

$\sin 2 \beta=\frac{i\left(G_{y x}-G_{x y}\right)}{\left[\left(G_{x x}-G_{y y}\right)^{2}+4 G_{y x} G_{x y}\right]^{1 / 2}}$,

where $x$ and $y$ denote the principal and second principal variance direction, respectively; $i$ stands for the unit of imaginary number; $\epsilon$ varies between -1 and 1: -1 for left-handed polarization; 0 for linear polarization; 1 for right-handed polarization. $\epsilon$ is compared among different spacecraft and exhibits a result very close to one another in frequency domain, i.e. polarization is coherent for $100 \mathrm{~km}$ distance. We obtain $\epsilon=0.187$ for the given frequency. Thus, the wave is elliptically right-hand polarized in the spacecraft frame. In the 
Table 1. Intervals, spacecraft frame frequencies, wavelengths, and the distances from the shock to Cluster 3 along IMF line of the foreshock wave observations.

\begin{tabular}{rrrrr}
\hline Date & $\mathrm{UT}$ & $f_{s / c}(\mathrm{mHz})$ & $\lambda(\mathrm{km})$ & $D\left(R_{E}\right)$ \\
\hline 3 Feb. 2002 & $04: 00-04: 35$ & 89.8 & 3926 & 0.9 \\
3 Feb. 2002 & $04: 00-04: 35$ & 113.3 & 3225 & 0.9 \\
11 Feb. 2002 & $22: 00-22: 35$ & 50.8 & 11781 & 16.8 \\
11 Feb. 2002 & $22: 00-22: 35$ & 160.2 & 1843 & 16.8 \\
12 Feb. 2002 & $10: 00-10: 35$ & 36.1 & 6283 & 4.7 \\
12 Feb. 2002 & $10: 00-10: 35$ & 156.2 & 3519 & 4.7 \\
12 Feb. 2002 & $12: 00-12: 35$ & 40.0 & 4282 & 2.5 \\
12 Feb. 2002 & $12: 00-12: 35$ & 101.6 & 2157 & 2.5 \\
12 Feb. 2002 & $14: 00-14: 35$ & 67.4 & 11780 & 1.4 \\
12 Feb. 2002 & $14: 00-14: 35$ & 398.4 & 1466 & 1.4 \\
13 Feb. 2002 & $16: 00-16: 35$ & 88.9 & 5237 & 0.5 \\
13 Feb. 2002 & $16: 00-16: 35$ & 308.6 & 1380 & 0.5 \\
16 Feb. 2002 & $07: 00-07: 35$ & 40.0 & 5542 & 8.4 \\
16 Feb. 2002 & $07: 00-07: 35$ & 164.1 & 1527 & 8.4 \\
18 Feb. 2002 & $08: 05-08: 40$ & 44.9 & 4284 & 14.1 \\
18 Feb. 2002 & $08: 05-08: 40$ & 312.5 & 901 & 14.1 \\
20 Feb. 2002 & $17: 00-17: 35$ & 55.7 & 4711 & 0.7 \\
20 Feb. 2002 & $22: 00-22: 35$ & 50.8 & 5234 & 9.6 \\
20 Feb. 2002 & $22: 00-22: 35$ & 101.6 & 4726 & 9.6 \\
21 Feb. 2002 & $22: 00-22: 35$ & 62.5 & 5234 & 14.5 \\
21 Feb. 2002 & $22: 00-22: 35$ & 246.1 & 3049 & 14.5 \\
26 Feb. 2002 & $20: 30-21: 05$ & 44.9 & 6283 & 0.1 \\
26 Feb. 2002 & $20: 30-21: 05$ & 269.5 & 755 & 0.1 \\
1 Mar. 2002 & $07: 00-07: 35$ & 47.9 & 1624 & 13.2 \\
1 Mar. 2002 & $07: 00-07: 35$ & 214.8 & 1162 & 13.2 \\
6 Mar. 2002 & $00: 30-01: 05$ & 36.1 & 6732 & 2.0 \\
6 Mar. 2002 & $00: 30-01: 05$ & 433.6 & 878 & 2.0 \\
7 Mar. 2002 & $05: 00-05: 35$ & 39.1 & 10472 & 5.5 \\
9 Mar. 2002 & $12: 00-12: 35$ & 30.3 & 7854 & 5.0 \\
9 Mar. 2002 & $14: 20-14: 55$ & 25.4 & 7250 & 5.0 \\
9 Mar. 2002 & $16: 00-16: 35$ & 27.3 & 9424 & 5.9 \\
\hline & & & &
\end{tabular}

plasma rest frame the sign of $\epsilon$ is changed, resulting from the reversal of propagation direction.

In summary, we have derived the following properties in the plasma rest frame: frequency $\sim 0.1 \times \Omega_{c p}$; wave number $\sim 0.1 \times \Omega_{c p} / V_{A}$ (also the wavelength of the order of $R_{E}$ ); phase velocity $\sim V_{A}$; propagation almost along the magnetic field $\left(\theta_{k B}=17^{\circ}\right)$; and elliptically left-handed polarization.

\section{Statistical study}

In the following statistical study we present various distributions of the foreshock wave properties: frequencies, wave numbers, phase velocities, propagation directions, and polarization, applying the analysis method introduced in the previous section. Intervals used for the statistical study are selected as follows. (A) The mission phase for $100 \mathrm{~km}$ spacecraft separation (from 3 February 2002 to 17 June 2002) is selected in order to resolve waves into small wavelengths up to $200 \mathrm{~km}$; (B) Bow shock crossings are identified by search-
Table 1. Continued.

\begin{tabular}{rcccc}
\hline Date & $\mathrm{UT}$ & $f_{s / c}(\mathrm{mHz})$ & $\lambda(\mathrm{km})$ & $D\left(R_{E}\right)$ \\
\hline 9 Mar. 2002 & $16: 00-16: 35$ & 175.8 & 25566 & 5.9 \\
10 Mar. 2002 & $17: 00-17: 35$ & 42.0 & 4284 & 4.7 \\
11 Mar. 2002 & $20: 00-20: 35$ & 46.9 & 6283 & 5.9 \\
11 Mar. 2002 & $20: 00-20: 35$ & 210.9 & 1163 & 5.9 \\
13 Mar. 2002 & $04: 30-05: 05$ & 34.2 & 3249 & 1.1 \\
16 Mar. 2002 & $13: 00-13: 35$ & 35.2 & 7853 & 1.1 \\
16 Mar. 2002 & $15: 00-15: 35$ & 30.3 & 7250 & 1.9 \\
16 Mar. 2002 & $15: 00-15: 35$ & 140.6 & 1083 & 1.9 \\
26 Mar. 2002 & $17: 50-18: 25$ & 29.3 & 11781 & 9.5 \\
26 Mar. 2002 & $17: 50-18: 25$ & 453.1 & 656 & 9.5 \\
27 Mar. 2002 & $07: 00-07: 35$ & 43.0 & 9425 & 4.1 \\
27 Mar. 2002 & $07: 00-07: 35$ & 335.9 & 972 & 4.1 \\
29 Mar. 2002 & $18: 00-18: 35$ & 114.3 & 1963 & 3.6 \\
29 Mar. 2002 & $18: 00-18: 35$ & 308.6 & 1107 & 3.6 \\
29 Mar. 2002 & $20: 30-21: 05$ & 55.7 & 2142 & 3.2 \\
29 Mar. 2002 & $20: 30-21: 05$ & 390.6 & 633 & 3.2 \\
2 Apr. 2002 & $04: 00-04: 35$ & 81.1 & 1273 & 16.2 \\
2 Apr. 2002 & $04: 00-04: 35$ & 160.2 & 1195 & 16.2 \\
3 Apr. 2002 & $16: 30-17: 05$ & 21.5 & 23561 & 16.3 \\
3 Apr. 2002 & $16: 30-17: 05$ & 457.0 & 1908 & 16.3 \\
27 Apr. 2002 & $02: 00-02: 35$ & 43.9 & 4099 & 2.7 \\
7 May 2002 & $23: 20-23: 55$ & 34.2 & 7854 & 3.5 \\
7 May 2002 & $23: 20-23: 55$ & 113.3 & 1505 & 3.5 \\
13 May 2002 & $14: 50-15: 25$ & 50.8 & 8567 & 3.0 \\
13 May 2002 & $14: 50-15: 25$ & 457.0 & 1400 & 3.0 \\
20 May 2002 & $04: 30-05: 05$ & 44.9 & 7854 & 1.8 \\
20 May 2002 & $06: 00-06: 35$ & 37.1 & 7249 & 12.3 \\
20 May 2002 & $08: 30-09: 05$ & 44.9 & 5890 & 4.0 \\
20 May 2002 & $08: 30-09: 05$ & 312.5 & 1616 & 4.0 \\
22 May 2002 & $11: 20-11: 55$ & 41.0 & 4097 & 6.0 \\
\hline & & & &
\end{tabular}

ing for the following characteristics: (a) An increase in the magnetic field magnitude when moving from the upstream solar wind to the magnetosheath (typically by a factor of 2 to 4); (b) a decrease in the ion bulk speed; (c) an increase in the ion density. The ion bulk speed $\sim 400 \mathrm{~km} / \mathrm{s}$ is also referred to in identifying the upstream region; (C) Moderately active fluctuation of the magnetic field is identified in the upstream region. For this purpose the variance of the fluctuation (defined in Sect. 2) $0.05 \leq \sigma^{2} \leq 0.40$ is imposed as a criterion; (D) The geometrical configuration is investigated and the intervals in the foreshock region are selected, applying the same method as described in the previous section.

For the selected intervals dominant frequencies are identified up to $0.5 \mathrm{~Hz}$ in the spacecraft frame. Sixty-one distinct waves from 36 events are selected under these criteria. Then wave numbers, rest frame frequencies, and phase velocities are investigated. Ellipticities are investigated as well. Table 1 lists all the intervals, frequencies in the spacecraft frame, wavelengths, and the distances $D$. The histogram of magnitude of wave number is displayed in Fig. 5, 


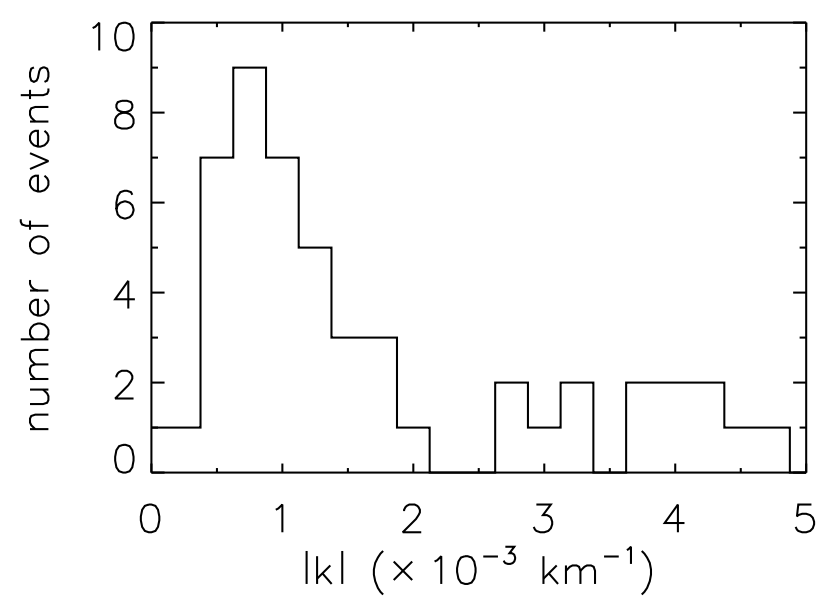

Fig. 5. Histogram of magnitude of wave number.

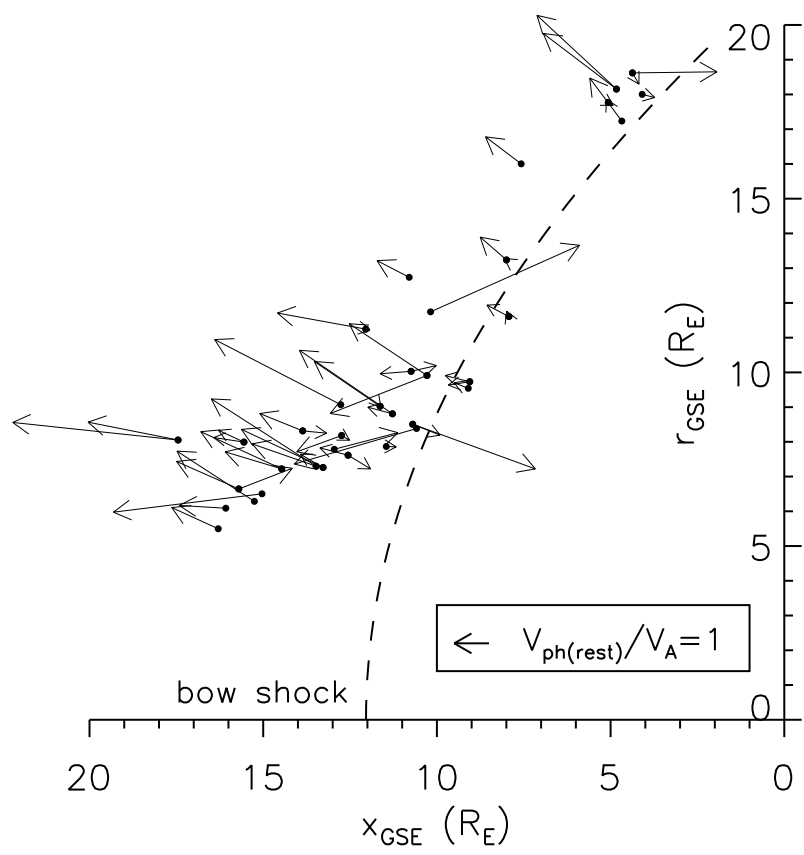

Fig. 6. Spatial distribution of wave phase velocities in the plasma rest frame plotted together with the location of wave observations, projected into the $x r$ plane in GSE $\left(r=\sqrt{y^{2}+z^{2}}\right)$. The phase velocities are normalized to the local Alfvén velocity. The dashed, curved line is a nominal bow shock.

which can be derived only from multi-point measurements. We find that most of wave numbers have a magnitude up to $2 \times 10^{-3} \mathrm{~km}^{-1}$. When normalized, this distribution exhibits a peak around $0.1 \times V_{A} / \Omega_{c p}$ (discussed later).

Figure 6 displays the spatial distribution of phase velocities in the plasma rest frame projected into the $x r$ plane in GSE $\left(r=\sqrt{y^{2}+z^{2}}\right)$. Small circles filled in black are the locations of wave observations and arrows starting from the circles are the phase velocities normalized to the local Alfvén velocity $V_{A}$. The dashed curved line represents a nominal bow shock for quiet solar wind conditions. We find that most of the waves propagate upstream and are more or less aligned with shock normal directions at various positions, near the $\mathrm{x}$ axis to near flank region. The phase velocities are of the order of the Alfvén velocity. Some of the waves, however, propagate downstream in the plasma rest frame. Such waves tend to have phase velocities smaller than the Alfvén velocity but a few cases show larger phase velocities near the shock.

The distributions of frequencies, wave numbers, propagation angles, and ellipticities are displayed in Fig. 7. Error bars are also present there. For scanning in the $k$ space we use a spherical grid with $75 \times 60 \times 60$ points in radial, azimuthal, and polar direction between $k_{\min }=0 \mathrm{~km}^{-1}$ and $k_{\max }=5 \times 10^{-3} \mathrm{~km}^{-1}$ for low-frequency waves up to $100 \mathrm{mHz}$ in the spacecraft frame, and between $0 \mathrm{~km}^{-1}$ and $25 \times 10^{-3} \mathrm{~km}^{-1}$ for the rest of frequencies. Mean $|\delta \boldsymbol{k}|$ is thus about $6.7 \times 10^{-5} \mathrm{~km}^{-1}$ for low-frequency waves and $3.3 \times 10^{-4} \mathrm{~km}^{-1}$ for high-frequency waves, and $\delta \theta_{k B}$ is $3^{\circ}$. Errors in the rest frame frequencies represent uncertainty in the Doppler shift, i.e. $\left|\delta \boldsymbol{k} \cdot \boldsymbol{V}_{s w}\right|+\left|\boldsymbol{k} \cdot \delta \boldsymbol{V}_{s w}\right|$. We use $\delta \boldsymbol{k}$ above and $\delta \boldsymbol{V}_{s w}$ which is calculated from mean absolute deviation of the ion velocity for Cluster 3 CIS-HIA. We do not assign error bars in the ellipticities, since the eigenvectors of spectral density matrix are uniquely determined (without any uncertainty). There are, however, various methods to investigate polarization parameters. Comparison among these methods may give the error estimate in the ellipticities. The left panel is the distribution of normalized frequencies $\omega_{\text {rest }} / \Omega_{c p}$ and magnitudes of normalized wave numbers $k V_{A} / \Omega_{c p}$. The signs of $\omega_{\text {rest }}$ and $\boldsymbol{k}$ are already changed, since most of the frequencies become negative after the Doppler shift correction. A magnified plot around $\omega / \Omega_{c p} \sim 0$ and $k V_{A} / \Omega_{c p} \sim 0.1$ is also embedded in the left panel. The dotted, straight line represents Alfvén waves propagating along the magnetic field with the relation $\omega / k=V_{A}$. Most of the waves are found at $\omega / \Omega_{c p} \sim 0.1$ and $k V_{A} / \Omega_{c p} \sim 0.1$, therefore, the major population of the waves propagates at speeds below and around up to the Alfvén velocity. Minor waves are found up to $\omega / \Omega_{c p} \sim 5$ and $k V_{A} / \Omega_{c p} \sim 1.2$. These waves are scattered in the $\omega-k$ distribution. Some of them follow $\omega / k=V_{A}$ and others deviate from it. The middle panel in Fig. 7 is the distribution of propagation angles $\theta_{k B}$ versus frequencies. The first major population is found at $\theta_{k B}>160^{\circ}$ and the second major population is found at $\theta_{k B}<30^{\circ}$. These two populations have small frequencies which correspond to the waves of $\omega / \Omega_{c p} \sim 0.1$ and $k V_{A} / \Omega_{c p} \sim 0.1$ in the left panel. Minor waves with relatively large frequencies and wave numbers have propagation angles perpendicular to the magnetic field rather than parallel/antiparallel. The right panel in Fig. 7 is the distribution of ellipticities, which are located between -0.5 and 0.2 . Major waves have a distribution centered slightly on the left-hand polarization side. Minor waves with larger magnitude of frequencies tend to be left-handed for positive frequencies (upstream propagation) and right-handed for negative frequencies (downstream propagation). 

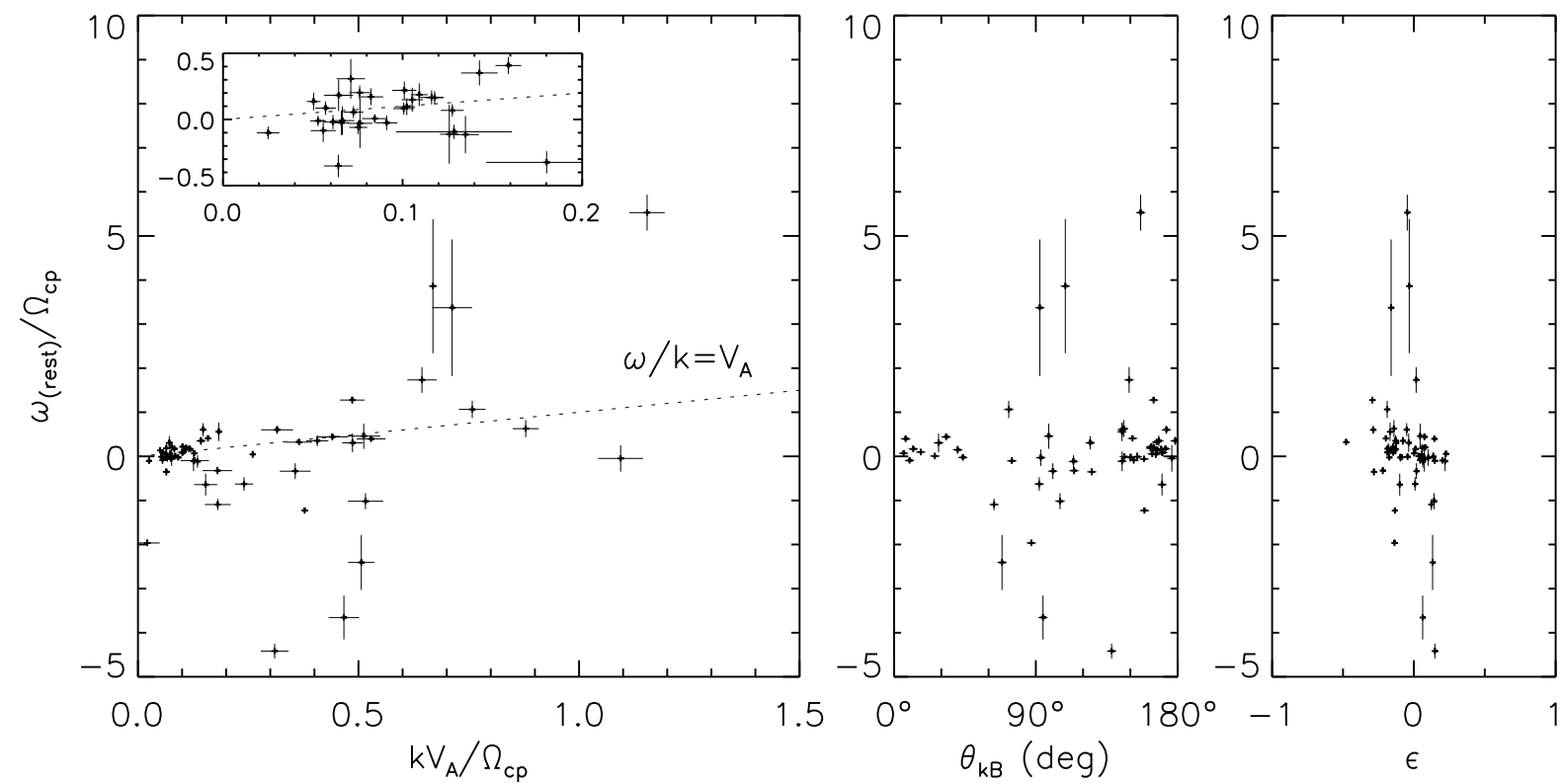

Fig. 7. Distribution of rest frame frequencies, wave numbers, propagation angles, and ellipticities in the plasma rest frame with error bar estimates. The small plot embedded in the left panel is a magnified plot of the frequencies and the wave numbers near origin. The dotted straight line in the left panel is a dispersion relation for the extended linear Alfvén waves. See Sect. 3 for calculation of the error bars.

In summary, most of the waves have frequencies $\sim 0.1 \times \Omega_{c p}$ and wave numbers $\sim 0.1 \times \Omega_{c p} / V_{A}$. They propagate upstream almost parallel/anti-parallel to the magnetic field, at phase velocities close to $V_{A}$ with left-hand polarization. We interpret that the dominant waves represent Alfvén waves because of good agreement in the phase velocity at low frequencies. Minor waves are also present, having propagation angles roughly perpendicular to the magnetic field at various phase velocities. Some minor waves agree with the dispersion relation of the Alfvén wave $\left(\omega / k=V_{A}\right)$ at larger frequencies and wave numbers.

We also examined if there is a possible relationship between frequencies or wave numbers and distances from the shock but they were relatively uniformly distributed and did not exhibit any clear signatures or organizations.

\section{Conclusions and discussion}

We have analyzed the foreshock waves using Cluster data and derived various properties, $\omega, \boldsymbol{k}, \boldsymbol{V}_{p h}, \theta_{k B}$, and $\epsilon$ in the plasma rest frame. The magnetic field data from all four Cluster spacecraft allowed us to investigate wave numbers and phase velocities experimentally and to compare them with the Alfvén velocity. In the statistical study the spatial distribution of phase velocities and the distributions of wave properties are presented.

Upstream propagation is dominating in the foreshock, as is expected from earlier case studies (Russell, 1971; Hoppe et al., 1981; Hoppe and Russell, 1983; Eastwood et al., 2002; Eastwood et al., 2003; Narita et al., 2003). We also identified rest frame frequencies $\sim 0.1 \times \Omega_{c p}$ and wave numbers $\sim 0.1 \times \Omega_{c p} / V_{A}$ (wavelengths of the order of $R_{E}$ ). These results confirm the ISEE observations (Hoppe et al., 1981; Hoppe and Russell, 1983) and imply propagation speeds close to Alfvén velocity. If we restrict the observations to propagation angle parallel/anti-parallel to the magnetic field at a frequency much smaller than the proton cyclotron frequency, we may conclude that the dominant wave in the foreshock is the Alfvén wave. Taking into account for the polarization analysis that waves are left-handed rather than righthanded, our results prefer the beam ion instability theory.

Some of the minor waves, despite larger wave numbers, had phase velocities close to the Alfvén velocity, whereas non-magnetohydrodynamic waves, such as the ion cyclotron resonant waves or the cold plasma waves, might be expected in this domain. These minor waves tend to propagate perpendicular to the magnetic field. The waves propagating along the magnetic field in the foreshock region have been widely studied and discussed, but the perpendicular propagation may be important as well in understanding the physical processes in this region. To identify the mode of such minor waves, the dispersion relation for each event will be investigated and compared with various theoretical models in a future paper. There we also plan to examine a possible association of the dispersion relations with the ion distribution functions.

Acknowledgements. The TU Braunschweig group was financially supported by the German Ministerium für Bildung und Forschung and the Deutsches Zentrum für Luft- und Raumfahrt under contract $500 \mathrm{OC} 0103$.

Topical Editor T. Pulkkinen thanks M. Goldstein and another referee for their help in evaluating this paper. 


\section{References}

Asbridge, J. R., Bame, S. J., and Strong, I. B.: Outward flow of protons from the Earth's bow shock, J. Geophys. Res., 73, 57775782, 1968.

Balikhin, M. A., Dudok de Wit, T., Alleyne, H. St. C. K., Woolliscroft, L. J. C., Walker, S. N., Krasnosel'skikh, V., MierJedrzejeowicz, W. A. C., and Baumjohann, W.: Experimental determination of the dispersion of waves observed upstream of a quasi-perpendicular shock, Geophys. Res. Lett., 24, 787-790, 1997a.

Balikhin, M. A., Woolliscroft, L. J. C., Alleyne, H. St. C., Dunlop, M., and Gedalin, M. A.: Determination of the dispersion of low frequency waves downstream of a quasiperpendicular collisionless shock, Ann. Geophys., 15, 143-151, 1997b.

Balogh, A., Carr, C. M., Acuña, M. H., Dunlop, M. W., et al.: The Cluster magnetic field investigation: overview of in-flight performance and initial results, Ann. Geophys., 19, 1207-1217, 2001.

Barnes, A.: Theory of generation of bow-shock-associated hydromagnetic waves in the upstream interplanetary medium, Cosmic Electrodyn., 1, 90-114, 1970.

Bendat, J. S. and Piersol, A. G.: Engineering applications of correlation and spectral analysis, John Wiley \& Sons, Inc., New York, 54-56, 1980.

Born, M. and Wolf, E.: Principles of optics, 6th Ed., Pergamon press, New York, 503-504, 550, 1980.

Burgess, D.: What do we really know about upstream waves?, Adv. Space Res., 20, 673-682, 1997.

Cairns, I. H., Fairfield, D. H., Anderson, R. R., Carlton, V. E. H., Paularena, K. I., and Lazarus, A. J.: Unusual location of Earth's bow shock on September 24-25, 1987: Mach number effects, J. Geophys. Res., 47-62, 1995.

Dudok de Wit, T., Krasnosel'skikh, V. V., Bale, S. D., Dunlop, M. W., Lühr, H., Schwartz, S. J., and Woolliscroft, L. J. C.: Determination of dispersion relations in quasi-stationary plasma turbulence using dual satellite data, Geophys. Res. Lett., 22, 26532656, 1995.

Eastwood, J. P., Balogh, A., Dunlop, M. W., Horbury, T. S., and Dandouras, I.: Cluster observations of fast magnetosonic waves in the terrestrial foreshock, Geophys. Res. Lett., 29, 2046-2049, 2002.

Eastwood, J. P., Balogh, A., and Lucek, E. A.: On the existence of Alfvén waves in the terrestrial foreshock, Ann. Geophys., 21, 1457-1465, 2003.

Escoubet, C. P., Fehringer, M., and Goldstein, M.: The Cluster mission, Ann. Geophys., 19, 1197-1200, 2001.

Fairfield, D. H.: Bow shock associated waves observed in the far upstream interplanetary medium, J. Geophys. Res., 74, 35413553, 1969.

Farris, M. H. and Russell, C. T.: Determining the standoff distance of the bow shock: Mach number dependence and use of models, J. Geophys. Res., 99, 17 681-17 689, 1994.

Fowler, R. A., Kotick, B. J., and Elliott, R. D.: Polarization analysis of natural and artificially induced geomagnetic micropulsations, J. Geophys. Res., 72, 2871-2883, 1967.

Fredericks, R. W.: A model for generation of bow-shock-associated upstream waves, J. Geophys. Res., 80, 7-17, 1975.

Gary, S. P.: Electromagnetic ion/ion instabilities and their consequences in space plasma: A review, Space Sci. Rev., 56, 373, 1991.

Glassmeier, K.-H., Motschmann, U., Dunlop, M., Balogh, A., Acuña, M. H., Carr, C., Musmann, G., Fornaçon, K.-H.,
Schweda, K., Vogt, J., Georgescu, E., and Buchert, S.: Cluster as a wave telescope - first results from the fluxgate magnetometer, Ann. Geophys., 19, 1439-1447, 2001, Correction, Ann. Geophys., 21, 1071, 2003.

Gosling, J. T., Asbridge, J. R., Bame, S. J., Paschmann, G., and Sckopke, N.: Observations of two distinct populations of bow shock ions in the upstream solar wind, Geophys. Res. Lett., 5, 957-960, 1978.

Greenstadt, E. W., Green, I. M., Inouye, G. T., Hundhausen, A. J., Bame, S. J., and Strong I. B.: Correlated magnetic field and plasma observations of the Earth's bow shock, J. Geophys. Res., 73, 51-60, 1968.

Greenstadt, E. W., Le, G., and Strangeway, R. J.: ULF waves in the foreshock, Adv. Space Res., 15, 71-84, 1995.

Hoppe, M. M., Russell, C. T., Frank, L. A., Eastman, T. E., and Greenstadt, E. W.: Upstream hydromagnetic waves and their association with backstreaming ion populations: ISEE 1 and 2 observations, J. Geophys. Res., 86, 4471-4492, 1981.

Hoppe, M. M. and Russell, C. T.: Plasma rest frame frequencies and polarizations of the low-frequency upstream waves: ISEE 1 and 2 observations, J. Geophys. Res., 88, 2021-2028, 1983.

Le, G. and Russell, C. T.: A study of coherence length of ULF waves in the Earth's foreshock, J. Geophys. Res., 95, $10703-$ 10706, 1990.

Merka, J., Szabo, A., Narock, T. W., King, J. H., Paularena, K. I., and Richardson, J. D.: A comparison of IMP 8 observed bow shock positions with model predictions, J. Geophys. Res., 108, (A2), doi:10.1029/2002JA009384, 2003.

Motschmann, U., Woodward, T. I., Glassmeier, K. H., and Dunlop, M. W.: Array Signal Processing Techniques, Proc. CLUSTER Workshop on Data Analysis Tools, edited by Glassmeier, K. H., Motschmann, U., and Schmidt, R., ESA SP-371, Paris, 79-86, 1995.

Motschmann, U., Woodward, T. I., Glassmeier, K. H., Southwood, D. J., and Pinçon, J. L.: Wavelength and direction filtering by magnetic measurements at satellite arrays: Generalized minimum variance analysis, J. Geophys. Res., 101, 4961-4965, 1996.

Narita, Y., Glassmeier, K.-H., Schäfer, S., Motschmann, U., Sauer, K., Dandouras, I., Fornaçon, K.-H., Georgescu, E., and Rème, H.: Dispersion analysis of ULF waves in the foreshock using cluster data and the wave telescope technique, Geophys. Res. Lett., 30, 1710-1713, 2003.

Paschmann, G., Sckopke, N., Bame, S. J., Asbridge, J. R., Gosling, J. T., Russell, C. T., and Greenstadt, E. W.: Association of lowfrequency waves with suprathermal ions in the upstream solar wind, Geophys. Res. Lett., 6, 209-212, 1979.

Pinçon, J. L. and Lefeuvre, F.: Local characterization of homogeneous turbulence in a space plasma from simultaneous measurement of field components at several points in space, J. Geophys. Res., 96, 1789-1802, 1991.

Pinçon, J. L. and Motschmann, U.: Multi-Spacecraft Filtering: General Framework, Analysis methods for multi-spacecraft data, edited by Paschmann, G. and Daly, P. W., ISSI Sci. Rep. SR-001, Bern, 65-78, 1998.

Rème, H., Aoustin, C., Bosqued, J. M., Dandouras, I., et al.: First multispacecraft ion measurements in and near the Earth's magnetosphere with the identical Cluster Ion Spectrometry (CIS) experiment, Ann. Geophys., 19, 1303-1354, 2001.

Russell, C. T., Childers, D. D., and Coleman Jr., P. J.: OGO 5 observations of upstream waves in interplanetary medium: Discrete wave packets, J. Geophys. Res., 76, 845-861, 1971. 
Russell, C. T.: Planetary Bow Shocks, in collisionless shocks in the heliosphere: reviews of current research, Geophysical Monograph 35, edited by Tsurutani, B. T. and Stone, R. G., Amer. Geophys. Union, Washington, D.C., 109, 1985.

Schwartz, S.: Shock and discontinuity normals, Mach numbers, and related parameters, Analysis methods for multi-spacecraft data, edited by Paschmann, G. and Daly, P. W., ISSI Sci. Rep. SR-001, Bern, 249-270, 1998.
Sentman, D. D., Edmiston, J. P., and Frank L. A.: Instabilities of low frequency, parallel propagating electromagnetic waves in the Earth's foreshock region, J. Geophys. Res., 86, 7487-7497, 1981.

Shue, J.-H., Chao, J. K., Fu, H. C., Russell, C. T., Song, P., Khurana, K. KI., and Singer, H. J.: A new functional form to study the solar wind control of the magnetopause size and shape, J. Geophys. Res., 102, 9497-9511, 1997. 